\title{
Cervical Chondrocutaneous Branchial Remnants
}

\author{
Daniel T. Ginat ${ }^{1} \cdot$ Daniel N. Johnson $^{2} \cdot$ Andrea Shogan $^{3} \cdot$ Nicole A. Cipriani $^{2}$
}

Received: 4 June 2017 / Accepted: 19 June 2017 / Published online: 6 July 2017

(C) Springer Science+Business Media, LLC 2017

\begin{abstract}
Cervical chondrocutaneous branchial remnants are rare congenital choristomas. These lesions contain a cartilage core surrounded by skin with adnexal structures and subcutaneous fat. Correspondingly, on ultrasound there is a tubular hypoechoic core surrounded by hyperechoic, while on CT there is central intermediate attenuation surrounded by fat attenuation tissues. These features are exemplified in this sine qua non radiology-pathology correlation article. Management includes complete surgical resection and evaluating for potential associated anomalies, such as other branchial apparatus anomalies, as well as cardiac anomalies.
\end{abstract}

Keywords Cervical chondrocutaneous branchial remnant $\cdot$ Choristoma $\cdot \mathrm{CT} \cdot$ Ultrasound

\section{History}

The patient presented with bilateral anterior lower neck skin tags at birth. The patient also has a history of recurrent bilateral serous otitis media during infancy and a ventricular septal defect. There is no known family history of such abnormalities.

Daniel T. Ginat

ginatd01@gmail.com

1 Department of Radiology, University of Chicago, $5841 \mathrm{~S}$ Maryland Avenue, Chicago, IL 60637, USA

2 Department of Pathology, University of Chicago, Chicago, IL, USA

3 Department of Surgery, Section of Otolaryngology, University of Chicago, Chicago, IL, USA

\section{Radiographic Features}

Ultrasound of the lower neck skin lesions showed hypoechoic cartilaginous centers and surrounding hyperechoic subcutaneous fat and skin (Fig. 1). There was no internal vascularity on Doppler. CT of the neck with contrast was also performed, which revealed tubular bilateral anterior lower neck lesions covered with skin and subcutaneous fat and an intermediate attenuation cartilage core that extends to the underlying to the underlying sternocleidomastoid muscles (Fig. 2).

\section{Diagnosis}

The patient underwent surgical resection of the skin lesions. During surgery cartilaginous remnants were observed to extend down to the sternocleidomastoid muscles. There was no evidence of any sort of fistula or tract. Histologic examination showed mature elastic cartilage cores covered by normal skin consisting of epidermis and dermis with adnexal structures and subcutaneous fat compatible with cervical chondrocutaneous branchial remnants (Fig. 3).

\section{Treatment}

Following complete resection of the bilateral cervical chondrocutaneous branchial remnants, there was no evidence of recurrence at 12 years follow up and no additional treatment for this was necessary. The patient did receive myringotomy tubes for the serous otitis media, which resolved during infancy. 

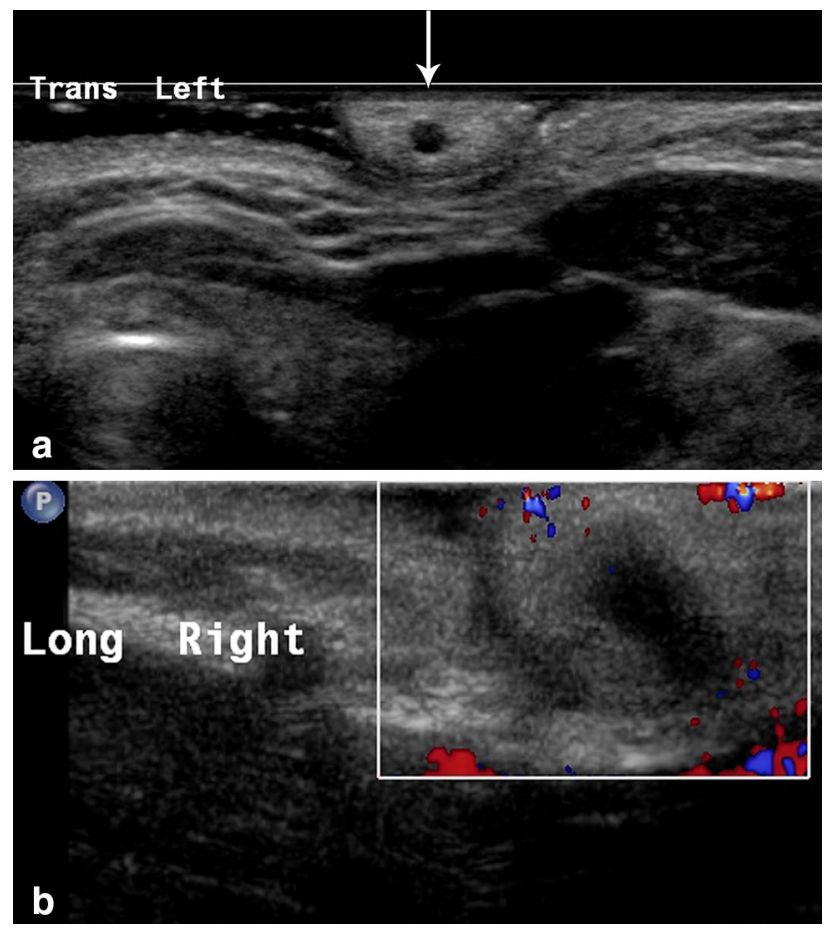

Fig. 1 Ultrasound image (a) shows one of the lesions in cross-section with a hypoechoic cartilaginous center and surrounding hyperechoic subcutaneous fat and skin (arrow). Doppler ultrasound image (b) along the length of the contralateral lesion shows that it is avascular

\section{Discussion}

Cervical chondrocutaneous branchial remnants are rare, benign, congenital choristomas [1-6]. These lesions are analogous to preauricular tags, but are located more inferiorly in the anterolateral neck. Although most often unilateral, chondrocutaneous branchial remnants are occasionally bilaterally, such as in this case. Some cases appear to be familial forms [1].

The remnants can contain either rod-shaped elastic or hyaline cartilage cores surrounded by normal skin and subcutaneous tissues with adnexa [1-3]. The presence of elastic cartilage suggests that the remnant is derived from the first branchial arch, while the presence of hyaline cartilage excludes an auricular origin and suggests an origin from the second or lower branchial arches [1].

Diagnostic imaging can be useful for delineating the lesions, in which the characteristic presence of the tubular cartilage that extends to the underlying sternocleidomastoid muscle. Thus, the imaging differential diagnosis is essentially limited to rare skin tags that may be
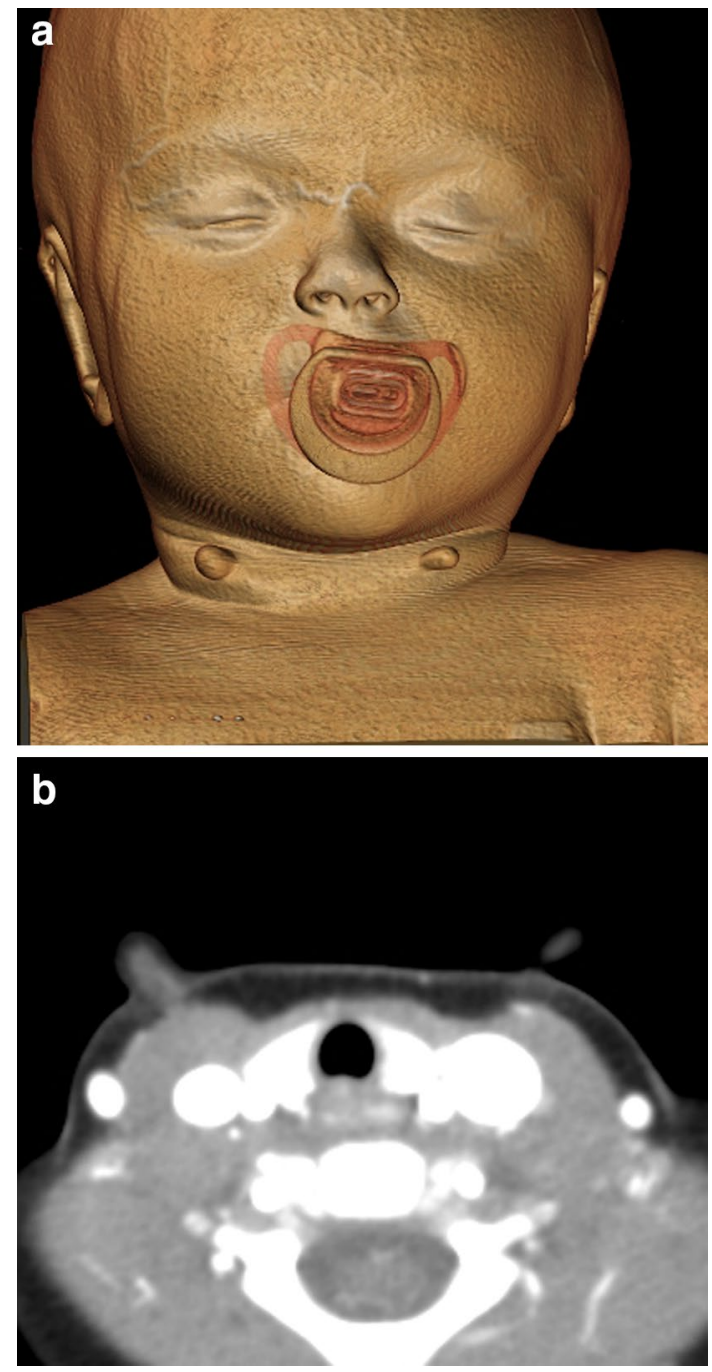

Fig. 2 3D (a) and axial (b) CT images show tubular bilateral anterior lower neck lesions covered with skin and subcutaneous fat and a central intermediate attenuation cartilage core that extends to the underlying to the underlying sternocleidomastoid muscles

associated with thyroglossal duct cysts, but these lack the cartilage core and are located closer to the midline. Imaging is recommended to evaluate for additional branchial apparatus anomalies and any associated cardiac or genitourinary anomalies, which have been reported in 11-76\% of cases [2,3].

The treatment of choice for cervical chondrocutaneous branchial remnants is early and complete surgical excision in order to obtain a histologic diagnosis and to achieve optimal cosmetic results $[2,4]$. 

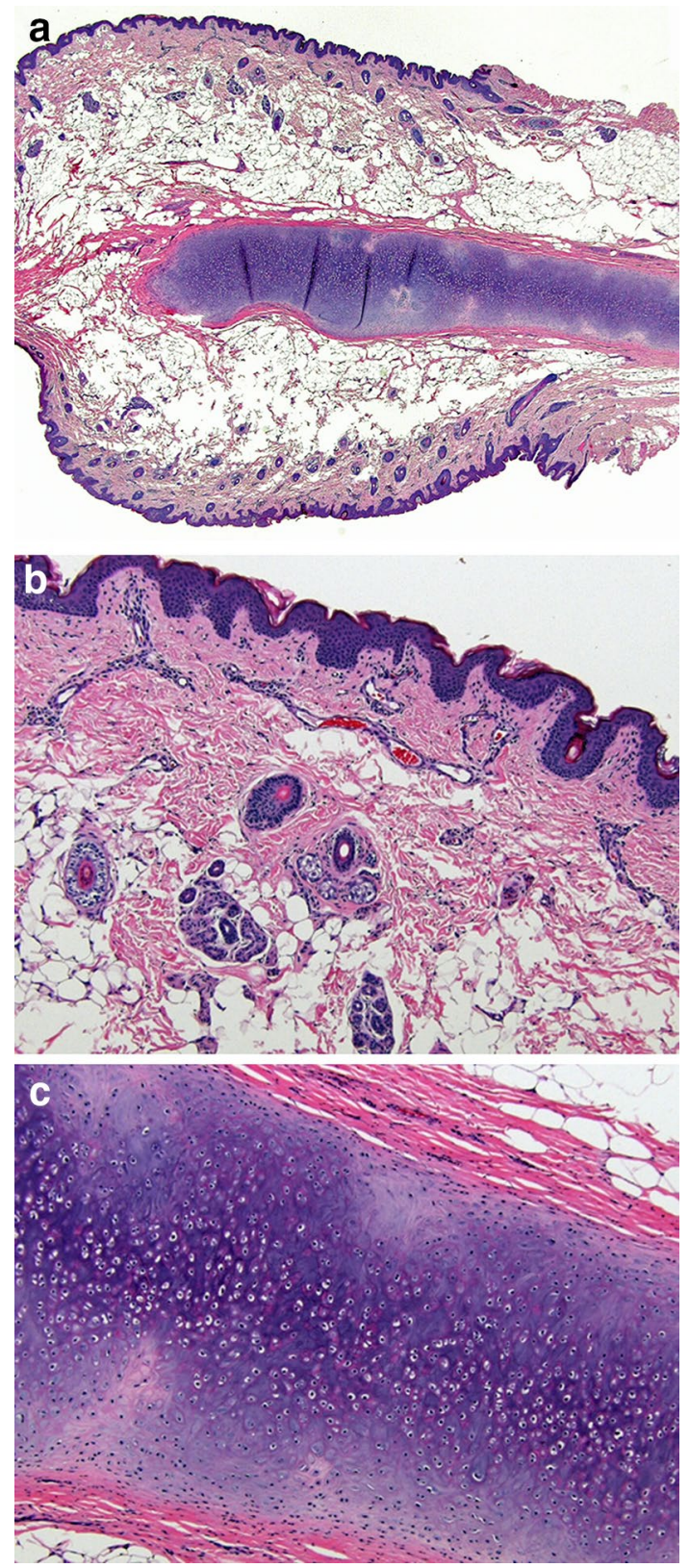

Fig. 3 Hematoxylin and eosin-stained histologic sections at 20x (a) and 100x (b and c) magnification show a rod-shaped mature elastic cartilage core covered by normal skin consisting of epidermis and dermis with adnexal structures and subcutaneous fat
Acknowledgements We are grateful for support received from the University of Chicago Office of Faculty Affairs through the Faculty Initiatives Fund for our Head and Neck Radiology-Pathology Trainee Conference, during which this case was presented.

\section{Compliance with Ethical Standards}

Conflict of interest The authors declare that they have no conflict of interest.

\section{References}

1. Pham Dang N, Chevaleyre A, Troude B, Mondié JM, Barthélémy I. Bilateral cervical chondrocutaneous remnants: a familial observation. Oral Maxillofac Surg. 2013;51:e288-e90.

2. Begovic N, Simic R, Vlahovic A, Kravljanac D, Djuricic S, Mijovic T. Cervical chondrocutaneous branchial remnants--report of 17 cases. Pediatr Otorhinolaryngol. 2014;78:1961-4.

3. Atlan G, Egerszegi EP, Brochu P, Caouette-Laberge L, Bortoluzzi P. Cervical chrondrocutaneous branchial remnants. Reconstr Surg. 1997;100:32-9.

4. Braun H, Hofmann T, Wolfgruber H, Anderhuber W, Beham A, Stammberger H. Case report of bilateral cervical chondrocutaneous branchial remnants. Pediatr Otorhinolaryngol. 2003;67:89-92.

5. Nasser HA, Iskandarani F, Berjaoui T, Fleifel S. A case report of bilateral cervical chondrocutaneous remnants with review of the literature. J Pediatr Surg. 2011;46:998-1000.

6. Chander B, Dogra SS, Raina R, Sharma C, Sharma R. Chondrocutaneous branchial remnants or cartilaginous choristoma: terminology, biological behavior and salience of bilateral cervical lesions. Patoloji Derg. 2014;30:195-200. 